\title{
From bench to bedside a comprehensive review of pancreatic cancer immunotherapy
}

\author{
Paul R. Kunk', Todd W. Bauer², Craig L. Slingluff ${ }^{3}$ and Osama E. Rahma ${ }^{1 *}$
}

\begin{abstract}
The incidence of pancreatic cancer has been increasing while its 5-year survival rate has not changed in decades. In the era of personalized medicine, immunotherapy has emerged as a promising treatment modality in a variety of malignancies, including pancreatic cancer. This review will discuss the unique pancreatic tumor microenvironment, including the cells and receptors that transform the pancreas from its normal architecture into a complex mix of suppressor immune cells and dense extracellular matrix that allows for the unrestricted growth of cancer cells. Next, we will highlight the recently completed immunotherapy clinical trials in pancreatic cancer. Finally, we will explore the on-going immunotherapy clinical trials and future directions of this engaging and changing field.
\end{abstract}

Keywords: Pancreatic cancer, Immunotherapy, Cancer vaccine, Immune checkpoint, Tumor Immunology

\section{Background}

Despite intensive research efforts to better understand its tumor microenvironment, the prognosis of pancreatic cancer remains dismal [1, 2]. The Pancreatic Cancer Action Network estimates that deaths from pancreatic cancer will be second only to lung cancer by 2020 [3]. Accordingly, novel treatment strategies for pancreatic cancer are desperately needed.

Immunotherapy is one of these novel strategies that has been under investigation in a variety of cancers. This review will focus on pancreatic cancer from an immune perspective, describing its immune microenvironment and the completed and ongoing clinical trials in this area.

\section{Review}

Pancreatic cancer from an immune perspective

Pancreatic cancer is unique from an immunological perspective. First, intratumoral effector T-cells are rare, in contrast to many other solid tumors for which infiltration of effector T-cells is often prominent $[4,5]$. Second, the $R A S$ oncogene drives an inflammatory program that establishes immune privilege in the pancreatic tumor

\footnotetext{
* Correspondence: OR3V@virginia.edu

'Department of Medicine, Division of Hematology-Oncology, University of Virginia Health System, UVA Box 800716, Charlottesville, VA 22908, USA Full list of author information is available at the end of the article
}

microenvironment (PTME) [6]. Third, pancreatic cancer is associated with a massive infiltration of immunosuppressive leukocytes into the tumor microenvironment $[4,5]$. Fourth, the development of pancreatic cancer is associated with a strong desmoplastic reaction that consists of multiple cell types, molecular factors, and extracellular matrix [7]. This dense desmoplastic stromal reaction is one of the hallmarks of pancreatic cancer and plays a vital role in promoting angiogenesis while evading from immune cells $[4,8,9]$. Studies have uncovered a rich communication between stellate cells (fibroblasts) and cancer cells $[4,8,9]$. The abundance of PDGF (platelet derived growth factor), fibronectin, proteoglycans and hyaluronic acid distorts the normal pancreatic architecture and transforms it into a complex, abnormal configuration of seemingly impenetrable walls [7]. Accordingly, this extensive stroma is not only a passive barrier for the immune system but rather interacts with cancer cells and participates in its progression and invasion [7].

It is useful to examine the immune cells and receptors in pancreatic cancer based on their role in the development of an immune response and their correlation with prognosis. There are two immunological processes that determine the immune response against cancer cells: the effector process and the suppressor process. These cell types are summarized in Table 1 and Fig. 1. 
Table 1 Cellular Microenvironment of Pancreatic Cancer

\begin{tabular}{llll}
\hline Cell & Role in pancreatic cancer & Relationship to outcome & Reference \\
\hline NK & deactivated & $\uparrow$ tumor stage and $\downarrow$ survival & {$[11-13]$} \\
CD8+ T-Cell & deactivated & $\uparrow$ tumor stage and $\downarrow$ survival & {$[5,8,13]$} \\
CD4+ Th1-Cell & $\downarrow$ & $\uparrow$ tumor stage and $\downarrow$ survival & {$[12-14]$} \\
TAM (M1) & $\downarrow$ & $\uparrow$ tumor stage & {$[4,12-14]$} \\
DC & deactivated & $\uparrow$ survival & {$[8,13,17,18]$} \\
MDSC & $\uparrow$ & $\downarrow$ survival & {$[5,12,16,24]$} \\
Mast Cell & $\uparrow$ & $\uparrow$ metastases & {$[9,13]$} \\
T-regs & $\uparrow$ & $\downarrow$ survival & {$[4,5,12,22,23]$} \\
TAM (M2) & $\uparrow$ & $\uparrow$ stage and $\downarrow$ survival & {$[4,12-14]$} \\
Fibroblast & $\uparrow$ & $\uparrow$ stage and $\downarrow$ survival & {$[12,13,25,27]$} \\
CD4 + Th2 Cell & $\uparrow$ & $\uparrow$ stage and $\downarrow$ survival & {$[5,9,12-14,16,25]$} \\
\hline
\end{tabular}

$\uparrow$ increase, $\downarrow$ decrease, DC dendritic cell, MDSC myeloid derived suppressor cell, NK natural killer cell, TAM tumor associated macrophage

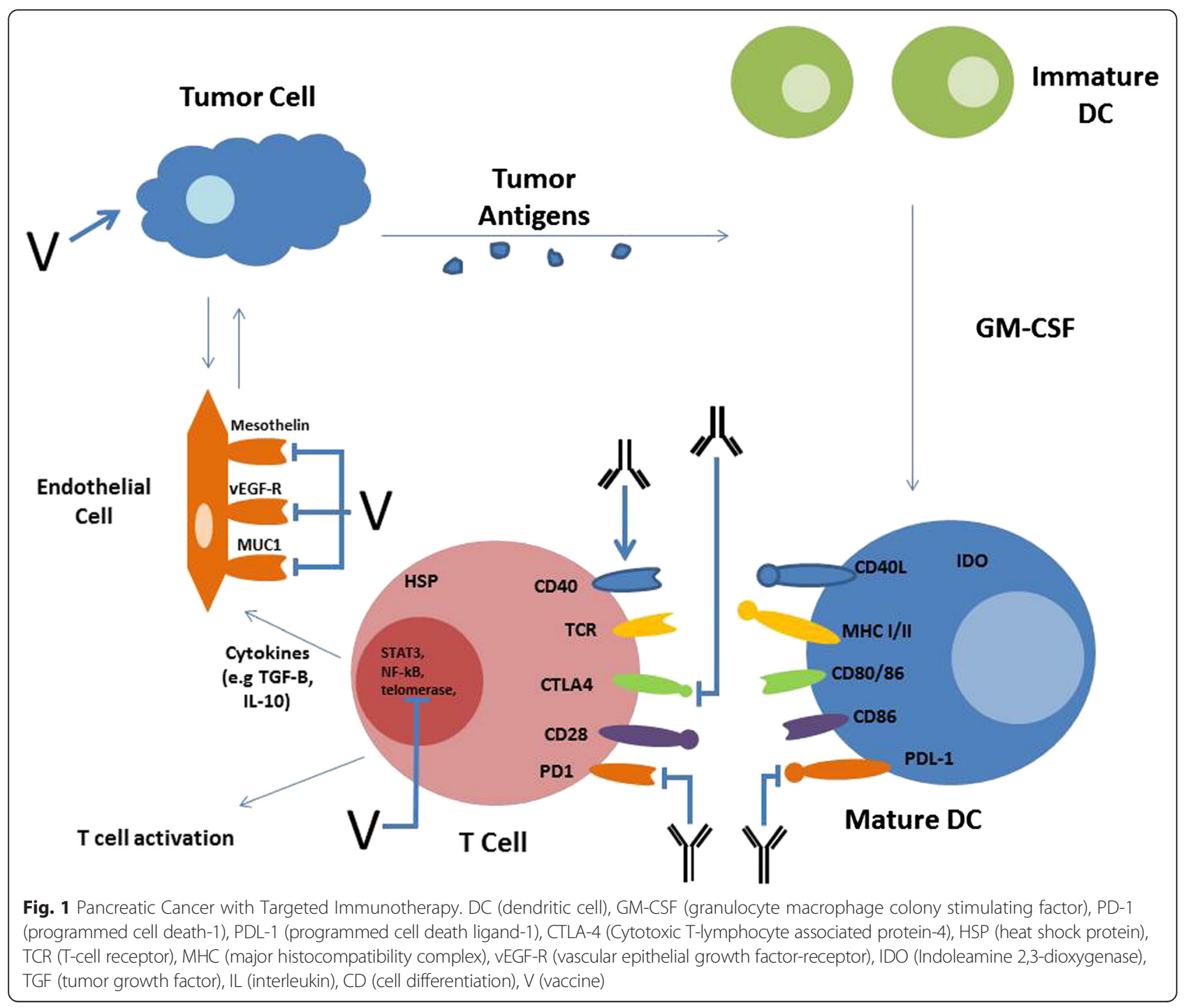




\section{Effector immune cells}

Natural killer cells (NK) An increased number of NK cells have been shown to be associated with a better prognosis in a small set of 13 patients with pancreatic cancer [10], presumably due to their role in recognition and elimination of cancer cells. However, NK cells are typically found in a limited number in pancreatic cancer and often in a deactivated form due to the lack of NKG2D, a cell surface receptor found to be upregulated in activated NK cells [11-13].

CD8 Cytotoxic and CD4 helper T-cells or tumor infiltrating lymphocytes (TILs) The presence of TILs in pancreatic cancer has been well described and it may represent the most important element in PTME $[8,9,14,5]$. Among these TILs, the memory (CD45RO) CD8 T-cells are thought to be the major anti-tumor effector cells and their density in resected pancreatic tumors was found to correlate with survival $[8,9]$. On the other hand, the role of CD4 T-cells is more complex. The Th1, the effector form, activates antigen presenting cells (APC) such as dendritic cells (DCs) while the ineffective form (Th2) plays a major role in tumor tolerance $[13,5]$. Although the presence of both CD8 and CD4 T-cells correlates with a better prognosis $[4,8,15]$, they are found in small numbers in the PTME, possibly due to effect of stroma and suppressor immune cells [15]. In addition, the number of CD8 effector T-cells decreases during the malignant transformation of pre-cancer cells $[5,8]$. Studies have shown that Th1 cells are found in disproportionately lower concentrations among pancreatic cancer cells than Th2, suggesting an ineffective immune response against tumor cells $[5,16]$.

Dendritic cells (DCs) The presence of DCs in the PTIM is essential in order to generate an anti-tumor immune response and, similar to TILs, is associated with a better prognosis in pancreatic cancer [8, 17, 18]. However, DCs are present in low numbers in the PTME and often in an immature form $[8,17,18]$, thus likely limiting their ability to present foreign antigens to T-cells.

\section{Co-stimulatory receptors and ligands}

CD40 Is a co-stimulatory molecule that is expressed on T-cells and binds to its ligand (CD40L) on APCs, leading to the activation of lymphocytes [13]. In a retrospective analysis of patients with pancreatic cancer, Unek et al., showed that the expression of CD40 in pancreatic cancer tissue samples correlates with a trend towards improved progression-free survival (PFS) and overall survival (OS) [19]. Accordingly, CD40 represents a promising target in pancreatic cancer as described below.
OX-40 (tumor necrosis factor receptor superfamily member 4) Is a member of the TNF receptor family found on T-cells and acts as a secondary co-stimulatory molecule as it requires other co-stimulatory molecules to be expressed first prior to its activation. The expression of OX-40 is found in high concentrations on activated T-cells. In pancreatic cancer increased levels of OX-40 was reported to correlate with better survival but this data needs to be validated on a larger scale [10].

4-1BB (tumor necrosis factor receptor superfamily member 9) Is also a member of the TNF receptor family and found on T-cells and NK cells. Upon interaction with its ligand 4-1BBL, it promotes $\mathrm{T}$ cell activation, particularly CD8 lymphocyte. 4-1BB also acts as a secondary co-stimulatory molecule, similar to $\mathrm{OX}-40[20,21]$. However, there is currently no data available regarding the role of $4-1 \mathrm{BB}$ in the PTIM.

\section{Suppressor immune cells}

Tumor associated macrophages (TAMs) These cells express the program cell death ligand (PD-L1) which is involved in immune suppression and T-cell apoptosis as described below. In PTIM, the presence of TAMs and it is association with poor outcomes and more frequent metastases has been well described $[4,13]$.

T-regulatory cells (CD4 + CD25 + FoxP3+) (T-regs) Tregs are other subsets of TILs known for their immunosuppressive activity through the release of cytokines including TGF- $\beta$ and IL-10. Based on few retrospective analyses, pancreatic tumors with low numbers of $\mathrm{T}$ regs were found to have a significantly better survival compared to tumors with high numbers [4, 22, 23].

Myeloid derived suppressor Cell (MDSCs) In pancreatic cancer, these cells were found in pre-malignant growths and increased in concentration as cancer cells grow suggesting a direct role in immune suppression and unrestricted cancer cell growth $[5,12,16,24]$. Interestingly, high concentration of MDSCs in the peripheral blood was associated with poor outcomes in patients with pancreatic cancer [16]. Whether this correlation exists in the PTIM remains to be determined.

Fibroblasts/pancreatic stellate cell Fibroblasts respond to a variety of molecules including CXCL12 (C-X-C motif chemokine 12) and produce VEGF (vascular endothelial growth factor) to stimulate angiogenesis in response to hypoxia or inflammation [7, 12, 13, 25]. In pancreatic cancer, the production of VEGF by fibroblasts is associated with cancer growth and worse prognosis 
$[26,27]$. Therefore, the role of fibroblasts in the PTME is currently under intense investigation.

Mast cells The role of mast cells in cancer has not been well-defined [9, 28, 29]. In pancreatic cancer, it has been suggested that low concentration of mast cells in the PTME correlates with increased survival [28] while increased concentration is associated with increased lymph node metastases based on retrospective analyses in small subsets of patients [29].

\section{Co-Inhibitory Receptors and Ligands}

Program-death (PD-1) Is an inhibitory receptor that belongs to the B7-receptor family and interacts with its ligand PD-L1 (B7-H1) to down regulate signals by Tcells, leading to the induction of apoptosis in activated T-cells [13]. PD-1 is expressed on progenitor T cells, activated T- and B-lymphocytes, NK cells, and myeloid cells [13]. Patients with pancreatic cancer with PD-L1 positive tumors have a significantly worse prognosis than patients with PD-L1 negative tumors [27]. The PD-1/ PD-L1 pathway is currently one of the most targeted pathways in cancer.

Cytotoxic T-lymphocyte associated protein-4 (CTLA-4) Is a co-inhibitory molecule found on $\mathrm{T}$ - lymphocytes that deactivates these cells to induce apoptosis in response to interaction with APCs. This negative feedback loop is a key to normal immune function to prevent over-stimulation of T-cells and damage to healthy cells but is stimulated inappropriately in pancreatic cancer to create a microenvironment that promotes cancer growth rather than its recognition. CTLA- 4 is overexpressed in pancreatic cancer cells and its overexpression was found to correlate negatively with survival in patients who underwent surgical resection [30].

CXCL12 (C-X-C motif chemokine ligand 12) Is a chemokine that is found in high concentration in pancreatic cancer and is involved in fibroblast migration and proliferation. The increased concentration of CXCL12 in the cancer microenvironment creates a network of dense stroma restricting immune cells migration and recognition of cancer antigens. Feig et al., showed that the inhibition of this chemokine resulted in increased T-cell infiltration into pancreatic cancer in murine models [27].

T-cell immunoglobulin and mucin-domain containing molecule 3 (TIM-3) Is a transmembrane protein that is involved in the regulation of Th1 lymphocytes. The interaction between TIM- 3 and CD4 Th1 cells has been studied in pancreatic cancer patients and found to correlate with tumor vascular invasion [31]. However, the role of TIM-3 needs to be further characterized in pancreatic cancer and other malignancies.

Soluble lymphocyte activation gene-3 (LAG-3) Is an important T-cell regulator that interacts with $\mathrm{MHC}$ class II molecules expressed on APCs. This interaction promotes activation and maturation of DCs but negatively regulates effector T-cells. LAG-3 has been shown to be necessary for T-regulatory cell activity and represents a novel target for therapy in pancreatic cancer [32].

Indoleamine 2,3-dioxygenase (IDO) Is an enzyme involved in cleaving tryptophan into kynurenine. Tryptophan is required for T-cell activation and kynurenine leads to T-regs differentiation and chemotaxis [33]. Increased levels of IDO, as seen in pancreatic cancer, creates a microenvironment devoid of effector T-cells but rich in immunosuppressive T-regs [34]. IDO inhibitors are currently under investigation in pancreatic cancer and other malignancies (NCT02048709 and NCT02077881).

Galectins (Gal-1, Gal-3 and Gal-9) Are immune modulating glycoproteins that are overexpressed in pancreatic cancer cells and thought to be involved in T-cell homeostasis. These glycoproteins have been shown to promote immune suppression in pancreatic cancer by promoting Th2 and T-reg transformation, restricting DC maturation and stimulating stellate cells $[35,36]$. Their association with survival in pancreatic cancer has been conflicting, with several studies showing increased concentration associated with decreased survival [37, 38], while others showed an association with improved outcomes [39].

B7-H3 Is a member of the B7 ligand family that can be induced in activated dendritic cells, monocytes, and $\mathrm{T}$ cells leading to decrease Th1 type response and cytokine production. B7-H3 expression was found to correlate with lymph node metastases and advanced pathologic stage in patients with pancreatic cancer [40]. The inhibition of B7-H3 increased CD8+ TILs and inhibited tumor growth in mice [40].

\section{Pancreatic cancer vaccines}

Cancer vaccines aim to stimulate the immune system against tumor cells by generating humoral and/or cellular immune responses. Many forms of cancer vaccines exist but generally they can be divided into synthetic and cellular-based vaccines.

\section{Synthetic vaccines (summarized in Table 2)}

Synthetic vaccines are typically made from whole protein or peptides that match a pre-determined antigen to induce a T- cell response. Despite multiple large trials 
Table 2 Completed immunotherapy clinical trials

\begin{tabular}{|c|c|c|c|c|c|c|c|}
\hline Treatment type & Target & N & Additional therapy & Cancer stage & Immunologic response & Clinical outcome & Ref \\
\hline \multirow[t]{22}{*}{ Peptide vaccines } & CEA & 23 & None & $\begin{array}{l}\text { Resected or } \\
\text { Metastatic }\end{array}$ & $\begin{array}{l}\uparrow \text { IFN- } \gamma \text { T cell response } \\
\text { by ELISPOT with increasing } \\
\text { vaccine dose }\end{array}$ & $37 \%$ survival at 32 months & {$[77]$} \\
\hline & $\begin{array}{l}\text { CEA + } \\
\text { MUC1 }\end{array}$ & 20 & None & Metastatic & NR & $\mathrm{mOS}$ of $7.3 \mathrm{~ms}$ & [78] \\
\hline & Gastrin 17 & 154 & None & Metastatic & $74 \%+$ ELISA & $\uparrow O S$ by $54 \%$ vs placebo $(p=0.03)$ & [51] \\
\hline & Gastrin 17 & 383 & None & Metastatic & $\begin{array}{l}\text { Correlation between } \\
\text { anti-gastrin17 titers } \\
\text { and OS }\end{array}$ & No benefit & [53] \\
\hline & Gastrin 17 & 30 & None & Metastatic & $67 \%+$ ELISA & $\uparrow O S(4$ to $7.2 \mathrm{~ms}$ if $+\operatorname{IR}(p<0.01)$ & [52] \\
\hline & $\begin{array}{l}\text { GVAX+ } \\
\text { Mesothelin }\end{array}$ & 90 & Cyclophosphamide & Metastatic & NR & $\uparrow \mathrm{OS}$ (4 to $6.2 \mathrm{~ms})$ & [63] \\
\hline & Hedgehog & 59 & Gemcitabine & Metastatic & NR & mOS $10 \mathrm{~ms}$ & [79] \\
\hline & KRAS & 23 & None & Resected & $85 \%+$ DTH & 10 year OS of $20 \%$ & [56] \\
\hline & KRAS & 48 & GM-CSF & $\begin{array}{l}\text { Resected(10) and } \\
\text { Metastatic (38) }\end{array}$ & $58 \%+\mathrm{DTH}$ & $\begin{array}{l}\uparrow O S(2 \text { to } 5.4 \mathrm{~ms} \text { if }+\mathrm{IR} \\
(p=0.0002)\end{array}$ & [54] \\
\hline & KRAS & 24 & GM-CSF & Resected & $11 \%+$ DTH & mOS 20.3 ms & [55] \\
\hline & KRAS & 39 & Gemcitabine & Resected & $47 \%+$ ELISpot & $\uparrow O S$ by 21.7 wks if $+\operatorname{IR}(p<0.01)$ & [57] \\
\hline & MUC1 & 16 & SB-AS adjuvant & Resected & $31 \%+\mathrm{DHT}$ & No benefit & {$[42]$} \\
\hline & MUC1 & 6 & $\begin{array}{l}\text { Incomplete } \\
\text { Freund's }\end{array}$ & Metastatic & $17 \%+$ ELISA & No benefit & [41] \\
\hline & Telomerase & 1062 & Gemcitabine & Metastatic & NR & No benefit & {$[47]$} \\
\hline & Telomerase & 520 & Gemcitabine & Metastatic & NR & No benefit & [48] \\
\hline & Telomerase & 48 & GM-CSF & Metastatic & $63 \%+$ DHT & $\begin{array}{l}\operatorname{mOS} \text { of } 4.3 \mathrm{~ms} \text { if }+\mathrm{IR} \\
(p<0.01)\end{array}$ & [50] \\
\hline & Telomerase & 178 & Gemcitabine & Metastatic & NR & No benefit & [49] \\
\hline & Trop-2 & 7 & None & Metastatic & NR & No benefit & {$[80]$} \\
\hline & VEGF & 607 & $\begin{array}{l}\text { Erlotinib + } \\
\text { Gemcitabine }\end{array}$ & Metastatic & NR & No benefit & [44] \\
\hline & VEGF & 535 & Gemcitabine & Unresectable & NR & No benefit alone & [46] \\
\hline & VEGF & 150 & Gemcitabine & Unresectable & NR & No benefit & [46] \\
\hline & $\begin{array}{l}\text { Wilm's } \\
\text { Tumor } \\
\text { gene-1 }\end{array}$ & 32 & Gemcitabine & Unresectable & $58 \%+\mathrm{DTH}$ & $\uparrow \mathrm{mOS}$ by $7 \mathrm{~ms}$ if $\mathrm{DHT}+(p<0.01)$ & [81] \\
\hline \multirow[t]{6}{*}{ Autologous: DC } & MUC-1 & 49 & Gemcitabine & Metastatic & $\downarrow 65 \%$ T-regs & $2 \mathrm{CR}, 5 \mathrm{PR}, 10 \mathrm{SD}$ & [59] \\
\hline & MUC1 & 17 & None & $\begin{array}{l}\text { Resected and } \\
\text { Unresectable }\end{array}$ & NR & $\mathrm{mOS}$ of $9 \mathrm{~ms}$ & [82] \\
\hline & MUC-1 & 20 & none & Metastatic & $\begin{array}{l}\text { Correlation between } \\
\text { CD38+ cells and OS }\end{array}$ & $\begin{array}{l}1 \text { pt had remission of lung mets, } \\
5 \text { had stable disease. mOS } 9.8 \text { mos }\end{array}$ & [58] \\
\hline & MUC-1 & 10 & None & Resected & No difference & $30 \%$ OS 4 years & [83] \\
\hline & MUC-1 & 2 & None & Metastatic & $\begin{array}{l}\uparrow 117 \% \text { CD8+ MUC-1 } \\
\text { specific cells }\end{array}$ & No benefit & {$[84]$} \\
\hline & $\begin{array}{l}\text { Wilm's } \\
\text { Tumor } \\
\text { gene-1 }\end{array}$ & 10 & Gemcitabine & Metastatic & $57 \%+$ DTH & $\uparrow O S$ if + DTH & [85] \\
\hline \multirow[t]{3}{*}{ Allogeneic } & GM-CSF & 60 & $5-\mathrm{FU}$ & Resected & $\uparrow$ mesothelin + ELISPOT & $\uparrow O S$ (53 \% to $76 \%$ if + IR) & [61] \\
\hline & GM-CSF & 14 & Adjuvant CRT & Resected & $21 \%+\mathrm{DTH}$ & DFS of $25 \mathrm{~ms}$ if $+\mathrm{R}$ & [60] \\
\hline & GM-CSF & 30 & Cyclophosphamide & Metastatic & ^Mesothelin ELISPOT & No benefit & [62] \\
\hline \multirow{3}{*}{$\begin{array}{l}\text { Adoptive cell } \\
\text { transfer }\end{array}$} & Mesothelin & 6 & None & Metastatic & NR & $33 \%$ with stable disease & [65] \\
\hline & MUC1 & 28 & None & $\begin{array}{l}\text { Resected (20) } \\
\text { and Unresectable (8) }\end{array}$ & $\begin{array}{l}\uparrow 10 \% \text { effector T-cells, } \\
\downarrow 5.7 \% \text { Tregs }\end{array}$ & $\begin{array}{l}\text { mOS } 5 \text { ms in unresectable, } \\
19 \% 3 \text { year OS in resectable }\end{array}$ & [64] \\
\hline & MUC1 & 20 & None & Unresected & $\uparrow C D 8+$ T-cells & mOS 9.8 ms, 1 year OS $20 \%$ & [58] \\
\hline
\end{tabular}


Table 2 Completed immunotherapy clinical trials (Continued)

\begin{tabular}{|c|c|c|c|c|c|c|c|}
\hline \multirow[t]{4}{*}{$\begin{array}{l}\text { Immune checkpoint } \\
\text { inhibitor }\end{array}$} & CD40 & 21 & Gemcitabine & $\begin{array}{l}\text { Unresected (7) and } \\
\text { Metastatic (20) }\end{array}$ & N/A & $\begin{array}{l}\uparrow m O S \text { by } 1.7 \text { ms vs gemcitabine } \\
\text { alone, } 1 \text { patient had complete } \\
\text { resolution of hepatic metasteses }\end{array}$ & [68] \\
\hline & CTLA-4 & 30 & GVAX & $\begin{array}{l}\text { Unresectable or } \\
\text { metastatic }\end{array}$ & N/A & $\begin{array}{l}\uparrow 1 \text { year OS by } 20 \% \text { compared } \\
\text { to GVAX alone }\end{array}$ & [75] \\
\hline & CTLA-4 & 27 & none & $\begin{array}{l}\text { Unresected (7) and } \\
\text { Metastatic (20) }\end{array}$ & N/A & $\begin{array}{l}1 \text { patient delayed regression } \\
\text { of hepatic metasteses }\end{array}$ & [70] \\
\hline & PD-L1 & 14 & none & Metastatic & $\mathrm{N} / \mathrm{A}$ & No benefit & [69] \\
\hline
\end{tabular}

$\uparrow$ increase, $\downarrow$ decrease, CR complete response, CRT chemoradiation, CTLA-4 Cytotoxic T-lymphocyte associated protein-4, DC dendritic cell, DTH delayed typed hypersensitivity, GM-CSF granulocyte macrophage colony stimulating factor, MDSC myeloid derived suppressor cell, mOS median overall survival, Ms months, N/A not applicable, NK natural killer cell, $N R$ not reported, $O S$ overall survival, $P D-L 1$, programmed cell death ligand-1, $P R$ partial response, $R$ response, $S D$ stable disease, TAM tumor associated macrophages, Wk weeks

targeting MUC1 [41-43], VEGF [44-46], telomerase [47-50] and gastrin-17 [51-53], none have shown a meaningful survival benefit. These trials, however, were able to show significant immune responses to the targeted antigens. Some trials using mutated RAS peptide vaccine alone [54-56] or in combination with gemcitabine [57] have shown clinical benefit. Importantly, this benefit was mainly seen in patients who demonstrated positive immune responses [54, 56, 57].

\section{Cellular-based vaccines (summarized in Table 2)}

Cellular-based vaccines use cancer cells (either whole cells or cell lysates) as the source of the antigens, allowing the immune system to utilize multiple antigens rather than a single epitope. Overall the results of these trials are encouraging; however, each included a small number of patients making it difficult for meaningful interpretation. Cellular based vaccines can be divided into autologous or allogeneic vaccines based on the source of the cells.

Autologous vaccines In this process, the patient's own dendritic cells are isolated and pulsed with a specific antigen before being re-infused back to the patient. Multiple studies have used this vaccination approach targeting variety of antigens. MUC1 is one of the most targeted antigens given its overexpression in pancreatic cancer and its association with tumor invasion and metastasis. The clinical outcome of this vaccination method was not impressive; however, an interesting correlation with immune biomarkers was identified such as increased CD38 (a marker for activated lymphocytes) [58] and decreased T-regs [59].

Allogeneic vaccine In this method of vaccination, a pancreatic cancer cell line is stimulated, usually with GM-CSF, in order to elicit an immune response when administered to patients with pancreatic cancer. A group at John Hopkins used this form of cancer vaccine (GVAX) in combination with 5-FU or chemoradiation in the adjuvant setting and showed an increase in PFS and OS in patients who developed an immune response against mesothelin $[60,61]$. The same group also investigated GVAX in the metastatic setting in combination with low dose cyclophosphamide, to deplete T-regs, demonstrating an increase in mesothelin specific T-cell response with no survival benefit [62]. More recently, GVAX was combined with a Listeria vaccine that expresses mesothelin in 90 patients with metastatic disease. The combination of these 2 vaccines showed an improved OS compared to the allogeneic vaccine alone (6.1 vs 3.9 months, $p=0.01$ ). Interestingly, patients who derived the most benefit of this combination were patients who received over 3 doses of the vaccine (9.7 vs 4.6 months, $p=0.01$ ) and patients who received at least 2 previous chemotherapy regimens (5.1 vs 3.7 months, $p=0.001$ ) [63]. This combination has currently a breakthrough designation by FDA while being investigated in patients with metastatic disease in a large ongoing clinical trial.

\section{Adoptive T- cell transfer (summarized in Table 2)}

In this approach the patient's T-cells are expanded and activated ex vivo then re-infused back to the patient. Based on the source and the method used for T-cell activation, adoptive T- cell transfer could be classified into: Tumor infiltrating lymphocytes (TILs), engineered $\mathrm{T}$ - cells that express a specific cancer T-cell receptor (TCR), and T- cells that express a chimeric antigen receptor (CAR). This methodology of immunotherapy had gained a lot of attention recently due to promising clinical outcomes in hematological malignancies. However, the efficacy of adoptive T- cell transfer remains to be determined in solid tumors including pancreatic cancer. Kawaoka et al., investigated MUC1-specific cytotoxic T lymphocytes (CTLs) in 28 patients and showed $19 \%$ 3-year survival in patients with resectable disease along with increased effector lymphocytes and decreased T-regs [64]. More recently, the University of Pennsylvania group presented their 
experience using autologous T- cells modified with a chimeric antigen receptor (CAR) that recognizes mesothelin in pancreatic cancer patients with refractory metastatic disease. Of the 6 patients treated 2 had stable disease with one patient had a decreased PET avidity of hepatic metastases. Overall, the treatment was well tolerated [65]. The carcinoembryonic antigen (CEA) is another attractive target in pancreatic cancer that is currently been explored in clinical trials (NCT01723306, NCT00004178 and NCT01212887). New generations of CAR therapies are under investigation with a focus on increasing their activities and specificities and decreasing their toxicities. The CAR T-cells efficacy could be enhanced by engineering the intracellular domain to contain co-stimulatory molecules such as $41 \mathrm{BB}$ and OX40 or combining CAR T-cells therapy with immune modulators such as cyclophosphamide in order to deplete T-regulatory cells (NCT02465983) or immune checkpoint inhibitors such as CTLA-4 and anti-PD1 antibodies.

\section{Immune checkpoint inhibitors and co-stimulatory agonists (summarized in Table 2)}

Immune checkpoint inhibitors represent a paradigm shift in cancer treatment due to their promising clinical activities in melanoma and other malignancies [66, 67]. However, a limited number of studies targeting these immune checkpoints have been completed in pancreatic cancer. The first study to investigate the costimulatory agonists in pancreatic cancer used CD40 agonist in combination with gemcitabine in 21 patients with locally advanced or metastatic disease. This study demonstrated promising outcomes with an improvement of median OS compared to gemcitabine alone and one patient having a complete resolution of his liver metastases [68]. In the area of immune checkpoint inhibitors, both CTLA-4 and PD-L1 inhibitors were investigated in patients with locally advanced or metastatic pancreatic cancer in 2 clinical trials. The clinical outcomes were disappointing, although, only small number of patients were treated on both trials $[69,70]$. To date, the only immune checkpoint inhibitor to show activity in pancreatic cancer is MEDI4736 (anti-PD-L1), which showed a PR rate of $8 \%$ in a preliminary analysis of this going trial [71]. While this suggests a response can be achieved with single immunotherapy, it remains dismal and maybe improved by combination therapy. This approach is currently being investigated in few ongoing trials as detailed in Table 3.

\section{Combination therapy}

Due to the lack of meaningful clinical benefits of cancer vaccines, the potential positive immunological effect of chemotherapy and radiation therapy, and the promising outcomes of immune checkpoint inhibitors, the focus has shifted towards combining these modalities. Gemcitabine, a standard chemotherapy that is used traditionally to treat pancreatic cancer, has been found to mediate immunological effects such as tumor associated antigen cross presentation by dendritic cells and the induction and expansion of cytotoxic $\mathrm{T}$ cells responses in addition to reduce the number of myeloid suppressor cells $[72,73]$. Radiation therapy can also increase the immunogenic properties of tumor cells by enhancing MHC class I expression, thereby increasing their vulnerability to CTLs. Another frequent effect of DNA damage inflicted by radiotherapy or chemotherapy is the increase in the expression of death receptors (in particular Fas/CD95 and TNF-related apoptosis-inducing ligand [TRAIL] receptors, enabling lysis of the tumor cells by Fas/CD95 ligand and TRAIL-positive immune effectors [74]. As detailed in Table 3, the majority of ongoing trials investigate a combination strategy of the immunotherapy with chemotherapy, radiation or both. Of interest are multiple trials targeting mesothelin and/or GVAX with chemoradiation and multiple immune checkpoint inhibitors combined with chemotherapy. Our group is currently investigating the immunological effect of the combination of chemoradiation and anti-PD-1 as a neoadjuvant treatment in patients with resectable or borderline resectable pancreatic cancer compared to neoadjuvant chemoradiation alone (NCT02305186). This neoadjuvant setting will allow investigators to study the effect of combination therapy on the tumor microenvironment. Another promising combination by the Hopkins group combined GVAX with anti-CTLA-4 and demonstrated a 1-year improvement in OS by $20 \%$ compared to GVAX and cyclophosphamide alone [75].

\section{Conclusion}

Despite the ongoing efforts outlined in this review, the prognosis of pancreatic cancer remains dismal. With the recent progress in cancer immunotherapy, there are glimmers of hope in new immune targets with more being identified each year. These advancements are moving from the bench to the bedside at a rapid pace, with the hope of translating into improvements in clinical outcomes. We believe that immunotherapy represents a promising modality in pancreatic cancer. However, there still remains much to be learned about the pancreatic immune microenvironment and its role in the immune escape of cancer cells. In order to develop an active strategy to enhance the immune response against pancreatic cancer that could be translated to a promising clinical outcome we must focus our efforts on increasing the density of the intratumoral effector T-cells; decreasing or inhibiting the 
Table 3 On-going immunotherapy clinical trials

\begin{tabular}{|c|c|c|c|c|c|c|}
\hline Treatment type & Phase & Target & $N$ & Additional therapy & Stage & Identifier \\
\hline Chimeric antibody & $1, \|$ & Ensituximab & 116 & None & Metastatic & NCT01040000 \\
\hline DNA vaccine & । & VEGFR-2 & 72 & None & Metastatic & NCT01486329 \\
\hline $\begin{array}{l}\text { Fungal vector } \\
\text { vaccine }\end{array}$ & $\|$ & RAS & 176 & Gemcitabine & Resected & NCT00300950 \\
\hline $\begin{array}{l}\text { Viral vector } \\
\text { vaccine }\end{array}$ & 1 & Small Pox Virus & 36 & None & Metastatic & NCT00574977 ${ }^{\mathrm{a}}$ \\
\hline \multirow[t]{11}{*}{ Allogeneic vaccine } & 1 & CEA & 48 & GM-CSF & Metastatic & NCT00028496 \\
\hline & 1 & Donor Lymphocyte & 37 & None & Metastatic & NCT00161187 \\
\hline & । & Dendritic cells & 12 & Poly-ICLC & Unresectable & NCT01677962 \\
\hline & $\|$ & GM-CSF & 60 & Cetuximab + Cyclophosphamide & Metastatic & NCT00305760 \\
\hline & $\|$ & GVAX & 56 & None & Metastatic & NCT00389610 \\
\hline & $\|$ & GVAX & 87 & Cyclophosphamide & Resectable & NCT0072744 \\
\hline & $\|$ & GVAX & 19 & $\begin{array}{l}\text { Cyclophosphamide, Radiation, } \\
\text { FOLFIRINOX }\end{array}$ & Resected & NCT01595321 \\
\hline & $\|$ & GVAX +/- Mesothelin & 93 & Cyclophosphamide & Metastatic & NCT01417000 \\
\hline & ॥ & GVAX +/- Mesothelin & 240 & $\begin{array}{l}\text { Gemcitabine, Capecitabine, 5-FU, } \\
\text { Irinotecan, Erlotinib or } \\
\text { Cyclophosphamide }\end{array}$ & Metastatic & NCT02004262 \\
\hline & $\|$ & IFN-a + GM-CSF & 14 & Cyclophosphamide & Metastatic & NCT00002773 \\
\hline & III & Virulizin & 400 & Gemcitabine +/- 5-FU & Metastatic & NCT00040092 \\
\hline \multirow{6}{*}{$\begin{array}{l}\text { Autologous } \\
\text { vaccine }\end{array}$} & । & CEA & 24 & None & Metastatic & NCT00004604 \\
\hline & I & CEA & 14 & None & Metastatic & NCT00027534 \\
\hline & I & CEA & 24 & Denileukin Diftitox & Metastatic & NCT00128622 \\
\hline & $\|$ & CEA & 48 & $\| \mathrm{L}-2$ & Metastatic & NCT01723306 \\
\hline & I & Dendritic Cells & 2 & $\begin{array}{l}\text { Gemcitabine + Stereotactic } \\
\text { Radiosurgery }\end{array}$ & Metastatic & NCT00547144 \\
\hline & $\|$ & $\mathrm{KLH}$ & 35 & Radiation & Metastatic & NCT00868114 \\
\hline \multirow[t]{11}{*}{ Immunotherapy } & I & $\mathrm{B} 7-\mathrm{H} 3$ & 93 & none & All & NCT01391143 \\
\hline & I & CD40 & 10 & Gemcitabine + nab-paclitaxel & Metastatic & NCT02588443 \\
\hline & I & CD40 & 10 & Gemcitabine & resected & NCT01456585 \\
\hline & $\|$ & CTLA-4 & 82 & None & Metastatic & NCT00112580 \\
\hline & । & CTLA-4 & 37 & Gemcitabine & Metastatic & NCT00556023 \\
\hline & I & CTLA-4 & 28 & Gemcitabine & Metastatic & NCT01473940 \\
\hline & $\|$ & CTLA-4 + GVAX & 92 & FOLFIRINOX & Metastatic & NCT01896869 \\
\hline & $\|$ & IDO & 98 & Gemcitabine + nab-paclitaxel & metastatic & NCT02077881 \\
\hline & 1 & IL-1-Ra & 13 & FOLFIRINOX & Metastatic & NCT02021422 \\
\hline & $|/| \mid$ & PD-1 & 56 & Capecitabine + Radiation & $\begin{array}{l}\text { Resectable and Borderline } \\
\text { Resectable }\end{array}$ & NCT02305186 \\
\hline & 1 & PD-L1 & 1038 & none & All & NCT01693562 \\
\hline \multirow[t]{6}{*}{ Peptide vaccine } & $1, \|$ & $\begin{array}{l}\text { Alpha }(1,3) \\
\text { galactosyltransferase }\end{array}$ & 7 & None & Metastatic & NCT00255827 \\
\hline & III & $\begin{array}{l}\text { Alpha }(1,3) \\
\text { galactosyltransferase }\end{array}$ & 280 & FOLFIRINOX & Locally Advanced & NCT01836432 \\
\hline & III & $\begin{array}{l}\text { Alpha }(1,3) \\
\text { galactosyltransferase }\end{array}$ & 722 & Gemcitabine, 5-FU, radiation & Resected & NCT01072981 \\
\hline & $\mathrm{l}, \mathrm{I}$ & CEA & 28 & None & Metastatic & NCT00529984 \\
\hline & I & $\mathrm{CEA}+\mathrm{MUC1}$ & 18 & None & Unresectable & NCT00669734 \\
\hline & । & hCG- $\beta$ & 36 & None & Metastatic & NCT00648102 \\
\hline
\end{tabular}


Table 3 On-going immunotherapy clinical trials (Continued)

\begin{tabular}{|c|c|c|c|c|c|}
\hline I & $h C G-\beta$ & 48 & None & Metastatic & NCT00709462 \\
\hline । & Heat Shock Protein & 16 & None & Resected & NCT00003025 \\
\hline$|/| \mid$ & Hedgehog & 122 & Gemcitabine & Metastatic & NCT01130142a \\
\hline I & Hedgehog & 21 & FOLFIRINOX & Unresectable & NCT01383538 \\
\hline । & MUC1 & 25 & None & Resected or Locally Advanced & NCT00008099 \\
\hline$|/| \mid$ & MUC5AC & 90 & Gemcitabine + nab-paclitaxel & Unresectable & NCT01834235 \\
\hline I & P53 & 12 & None & Unresectable & NCT01191684 \\
\hline$\|$ & P53 + RAS & 70 & $\mathbb{I L}-2$ & Metastatic & NCT00019084 \\
\hline I & RAS & 7 & None & Metastatic & NCT00006387 \\
\hline । & RAS & 33 & None & Metastatic & NCT00019006 ${ }^{\mathrm{a}}$ \\
\hline III & Telomerase & 1110 & Capecitabine + Gemcitabine & Metastatic & NCT00425360 \\
\hline । & TGF- $\beta$ & 168 & Gemcitabine & Resectable and unresectable & NCT01373164 \\
\hline I & Trophoblast glycoprotein & 44 & None & Metastatic & NCT00056537 \\
\hline$|/| \mid$ & VEGF & 17 & Gemcitabine & Unresectable & NCT00655785 \\
\hline । & VEGFR-2 & 21 & Gemcitabine & Metastatic & NCT00622622 \\
\hline
\end{tabular}

5-FU 5-flurouracil, CEA Carcinoembryonic antigen, CTLA-4 Cytotoxic T-lymphocyte associated protein-4, FOLFIRINOX folinic acid, fluorouracil, irinotecan, oxaliplatin, GM-CSF granulocyte macrophage colony stimulating factor, $h C G$ Human chorionic gonadotropin, IDO indoleamine 2,3-dioxygenase, IFN interferon, IL-2 interleukin-2, $K L H$ keyhole limpet hemocyanin, MUC mucin, $N$ number, $P D$ programmed death, $P D-L$ programmed death ligand, Poly-ICLC carboxymethylcellulose, polyinosinic-polycytidylic acid, and poly-L-lysine double-stranded RNA, TGF Transforming growth factor, VEGF Vascular endothelial growth factor, VEGF-R Vascular endothelial growth factor receptor aStudy listed as complete but results not published

immunosuppressive cells and receptors; and understanding the role of the stromal reaction and its interaction with pancreatic cancer immune microenvironment. Indeed, the recent encouraging data of patients with mismatch-repair deficient colorectal cancer responding to pembrolizumab requires further investigation, particularly as it may be relevant for a small number of patients with pancreatic cancer [76]. Improved response rates and survival benefits may be achieved by using combination therapies; identifying novel biomarkers in order to select the group of patients who may drive the most benefit of cancer immunotherapy; and implementing novel clinical trials designs that allow for tumor samples collection in order to understand the mechanism of action and resistance of pancreatic cancer to immunotherapy.

\footnotetext{
Abbreviations

$\uparrow$ : increase; $\downarrow$ : decrease; 4-1BB: Tumor Necrosis Factor Receptor Superfamily Member 9; APC: antigen presenting cell; CD: cell differentiation; CR: complete response; CRT: chemoradiation; CTLA-4: Cytotoxic T-lymphocyte associated protein-4; CXCL12: C-X-C motif chemokine 12; DC: dendritic cell; DTH: delayed typed hypersensitivity; ELISA: enzyme linked immunosorbent assay; FOLFIRINOX: folinic acid, fluorouracil, irinotecan, oxaliplatin; Gal: Galectins; GM-CSF: granulocyte macrophage colony stimulating factor; HSP: heat shock protein; IDO: Indoleamine 2,3-dioxygenase; IFN-ү: interferongamma; IL: interleukin; LAG-3: Soluble Lymphocyte Activation Gene-3; MDSC: myeloid derived suppressor cell; MHC: major histocompatibility complex; mOS: median overall survival; ms: months; NA: not applicable; NK: natural killer cell; NR: not reported; OS: overall survival; OX-40: Tumor Necrosis Factor Receptor Superfamily Member 4; PD-1: programmed cell death-1; PDGF: platelet derived growth factor; PD-L1: programmed cell death-ligand 1; PR: partial response; PTME: pancreatic tumor microenvironment; R: response; SD: stable disease; TAM: tumor associated macrophages; TCR: T-cell receptor; TGF: tumor growth factor; TIL: tumor infiltrating lymphocyte; TIM-3: T-cell Immunoglobulin and mucin-domain containing molecule 3;
}

vEGF: vascular epithelial growth factor; vEGF-R: vascular epithelial growth factor-receptor.

\section{Competing interests}

Paul Kunk has no conflicts of interest to disclose.

Osama Rahma is supported in part by a research grant from Investigatory-Initiated Studies Program of Merck, Sharp \& Dohme Corp.

Todd Bauer is has equity ownership of iTi Health, Inc. and is a consultant for Hemoshear, LLC.

Craig Slingluff receives funding from NIH but not related to the topic of this review.

\section{Authors' contributions}

PK collected the data and drafted the manuscript. OR revised the manuscript. TB and CS provided additional revisions for the manuscript. All authors read and approved the final manuscript.

\section{Authors' information}

PK is currently an Oncology Fellow at the University of Virginia (UVA) with interest in gastrointestinal malignancy. Awarded the Farrow Oncology Fellowship Award, he is currently studying the immune profile of cholangiocarcinoma and is gaining expertise in the field of hepatobiliary cancer and immunotherapy. OR is an Assistant Professor in the Division of Hematology/Oncology at UVA. During his previous training at the National Cancer Institute, he was involved in conducting cancer vaccine clinical trials and the development of immune checkpoint inhibitors. As a member of the Emily Couric Cancer Center and in collaboration with other investigators, his main focus is to study hepatobiliary cancers and to identify potential targets and immune biomarkers that may correlate with outcomes. He is currently a Principal Investigator on many immunotherapy clinical trials in $\mathrm{Gl}$ cancers and is the lead physician of the $\mathrm{Gl}$ Oncology team. TB is an Associate Professor of Surgery in the Division of Surgical Oncology, with emphasis on hepatobiliary tumors. Receipt of several awards for clinical and research excellence, his research focus is the immune relationship to pancreatic cancer and early detection of pancreatic cancer. He is the director of the High-Risk Pancreatic Cancer Clinic and a Principal Investigator in many clinical trials in Gl cancers. CS is a Professor of Surgery in the Division of Surgical Oncology and a leading expert in the immune profile and immunotherapy of melanoma. He is a Principal Investigator in several clinical trials in melanoma. 


\section{Acknowledgements}

No additional acknowledgements other than those listed as authors. There were no sources of funding for this review.

\section{Author details}

'Department of Medicine, Division of Hematology-Oncology, University of Virginia Health System, UVA Box 800716, Charlottesville, VA 22908, USA. ${ }^{2}$ Department of Surgery, Division of Hepatobiliary Surgery, University of Virginia Health System, Charlottesville, VA, USA. ${ }^{3}$ Department of Surgery, Division of Surgical Oncology, University of Virginia Health System, Charlottesville, VA, USA.

Received: 22 October 2015 Accepted: 16 February 2016 Published online: 15 March 2016

\section{References}

1. Jemal A, Siegel R, Xu J, Ward E. Cancer statistics, 2010. CA Cancer J Clin. 2010;60(5):277-300.

2. Yadav D, Lowenfels $A B$. The epidemiology of pancreatitis and pancreatic cancer. Gastroenterology. 2013;144(6):1252-61.

3. Rahib L, Smith BD, Aizenberg R, Rosenzweig AB, Fleshman JM, Matrisian LM. Projecting cancer incidence and deaths to 2030: the unexpected burden of thyroid, liver, and pancreas cancers in the United States. Cancer Res. 2014; 74(11):2913-21.

4. Ino $Y$, Yamazaki-Itoh $R$, Shimada K, Iwasaki M, Kosuge $T$, Kanai $Y$, et al. Immune cell infiltration as an indicator of the immune microenvironment of pancreatic cancer. Br J Cancer. 2013;108(4):914-23.

5. Fridman WH, Pages F, Sautes-Fridman C, Galon J. The immune contexture in human tumours: impact on clinical outcome. Nat Rev Cancer. 2012;12(4): 298-306.

6. Collins MA, Bednar F, Zhang Y, Brisset JC, Galban S, Galban CJ, et al. Oncogenic Kras is required for both the initiation and maintenance of pancreatic cancer in mice. J Clin Invest. 2012;122(2):639-53.

7. Feig C, Gopinathan A, Neesse A, Chan DS, Cook N, Tuveson DA. The pancreas cancer microenvironment. Clin Cancer Res. 2012;18(16):4266-76.

8. Fukunaga A, Miyamoto M, Cho Y, Murakami S, Kawarada Y, Oshikiri T, et al. CD8+ tumor-infiltrating lymphocytes together with CD4+ tumor-infiltrating lymphocytes and dendritic cells improve the prognosis of patients with pancreatic adenocarcinoma. Pancreas. 2004;28(1):e26-31.

9. Protti MP, De Monte L. Immune infiltrates as predictive markers of survival in pancreatic cancer patients. Front Physiol. 2013;4:210.

10. Davis M, Conlon K, Bohac GC, Barcenas J, Leslie W, Watkins L, et al. Effect of pemetrexed on innate immune killer cells and adaptive immune $T$ cells in subjects with adenocarcinoma of the pancreas. J Immunother. 2012;35(8):629-40.

11. Duan X, Deng L, Chen X, Lu Y, Zhang Q, Zhang K, et al. Clinical significance of the immunostimulatory MHC class I chain-related molecule A and NKG2D receptor on NK cells in pancreatic cancer. Med Oncol. 2011;28(2):466-74.

12. Sideras K, Braat H, Kwekkeboom J, van Eijck CH, Peppelenbosch MP, Sleijfer S, et al. Role of the immune system in pancreatic cancer progression and immune modulating treatment strategies. Cancer Treat Rev. 2014:40(4):513-22.

13. Wachsmann MB, Pop LM, Vitetta ES. Pancreatic ductal adenocarcinoma: a review of immunologic aspects. J Investig Med. 2012;60(4):643-63.

14. Disis ML. Immune regulation of cancer. J Clin Oncol. 2010;28(29):4531-8.

15. Vonderheide RH, Bayne LJ. Inflammatory networks and immune surveillance of pancreatic carcinoma. Curr Opin Immunol. 2013;25(2):200-5.

16. Gabitass RF, Annels NE, Stocken DD, Pandha HA, Middleton GW. Elevated myeloid-derived suppressor cells in pancreatic, esophageal and gastric cancer are an independent prognostic factor and are associated with significant elevation of the Th2 cytokine interleukin-13. Cancer Immunol Immunother. 2011;60(10):1419-30.

17. Yamamoto $T$, Yanagimoto H, Satoi S, Toyokawa H, Yamao J, Kim S, et al. Circulating myeloid dendritic cells as prognostic factors in patients with pancreatic cancer who have undergone surgical resection. J Surg Res. 2012; 173(2):299-308.

18. Tjomsland V, Spangeus A, Sandstrom P, Borch K, Messmer D, Larsson M. Semi mature blood dendritic cells exist in patients with ductal pancreatic adenocarcinoma owing to inflammatory factors released from the tumor. PLoS One. 2010;5(10):e13441.

19. Unek T, Unek IT, Agalar AA, Sagol O, Ellidokuz H, Ertener O, et al. CD40 expression in pancreatic cancer. Hepatogastroenterology. 2013;60(128): 2085-93.
20. Moran AE, Kovacsovics-Bankowski M, Weinberg AD. The TNFRs OX40, 4-1BB, and CD40 as targets for cancer immunotherapy. Curr Opin Immunol. 2013; 25(2):230-7

21. Cheuk AT, Mufti GJ, Guinn BA. Role of 4-1BB:4-1BB ligand in cancer immunotherapy. Cancer Gene Ther. 2004;11(3):215-26.

22. Ikemoto $T$, Yamaguchi $T$, Morine $Y$, Imura $S$, Soejima $Y$, Fujii $M$, et al. Clinical roles of increased populations of Foxp3 + CD4 $+T$ cells in peripheral blood from advanced pancreatic cancer patients. Pancreas. 2006;33(4):386-90

23. Yamamoto $T$, Yanagimoto H, Satoi S, Toyokawa H, Hirooka S, Yamaki S, et al. Circulating CD4 + CD25+ regulatory $T$ cells in patients with pancreatic cancer. Pancreas. 2012;41(3):409-15.

24. Zhao F, Obermann S, von Wasielewski R, Haile L, Manns MP, Korangy F, et al. Increase in frequency of myeloid-derived suppressor cells in mice with spontaneous pancreatic carcinoma. Immunology. 2009;128(1):141-9.

25. De Monte L, Reni M, Tassi E, Clavenna D, Papa I, Recalde H, et al. Intratumor T helper type 2 cell infiltrate correlates with cancer-associated fibroblast thymic stromal lymphopoietin production and reduced survival in pancreatic cancer. J Exp Med. 2011;208(3):469-78.

26. Abdollahi A, Schwager C, Kleeff J, Esposito I, Domhan S, Peschke P, et al. Transcriptional network governing the angiogenic switch in human pancreatic cancer. Proc Natl Acad Sci U S A. 2007;104(31):12890-5.

27. Feig C, Jones JO, Kraman M, Wells RJ, Deonarine A, Chan DS, et al. Targeting CXCL12 from FAP-expressing carcinoma-associated fibroblasts synergizes with anti-PD-L1 immunotherapy in pancreatic cancer. Proc Natl Acad Sci U S A. 2013;110(50):20212-7.

28. Strouch MJ, Cheon EC, Salabat MR, et al. Crosstalk between mast cells and pancreatic cancer cells contributes to pancreatic tumor progression. Clinical cancer research : an official journal of the American Association for Cancer Research. 2010;16(8):2257-65.

29. Esposito I, Menicagli M, Funel N, et al. Inflammatory cells contribute to the generation of an angiogenic phenotype in pancreatic ductal adenocarcinoma. J Clin Pathol. 2004;57(6):630-6.

30. Loos M, Hedderich DM, Ottenhausen M, Giese NA, Laschinger M, Esposito I, et al. Expression of the costimulatory molecule B7-H3 is associated with prolonged survival in human pancreatic cancer. BMC Cancer. 2009;9:463-2407-9-463.

31. Tong D, Zhou Y, Chen W, Deng Y, Li L, Jia Z, et al. T cell immunoglobulinand mucin-domain-containing molecule 3 gene polymorphisms and susceptibility to pancreatic cancer. Mol Biol Rep. 2012;39(11):9941-6.

32. Wang-Gillam A, Plambeck-Suess S, Goedegebuure P, Simon PO, Mitchem JB, Hornick JR, et al. A phase I study of IMP321 and gemcitabine as the frontline therapy in patients with advanced pancreatic adenocarcinoma. Invest New Drugs. 2013;31(3):707-13.

33. Fallarino F, Grohmann U, You S, McGrath BC, Cavener DR, Vacca C, et al. The combined effects of tryptophan starvation and tryptophan catabolites down-regulate $T$ cell receptor zeta-chain and induce a regulatory phenotype in naive T cells. J Immunol. 2006;176(11):6752-61.

34. Witkiewicz A, Williams TK, Cozzitorto J, Durkan B, Showalter SL, Yeo CJ, et al. Expression of indoleamine 2,3-dioxygenase in metastatic pancreatic ductal adenocarcinoma recruits regulatory $T$ cells to avoid immune detection. J Am Coll Surg. 2008;206(5):849-54. discussion 854-6.

35. Cooper D, llarregui JM, Pesoa SA, Croci DO, Perretti M, Rabinovich GA. Multiple functional targets of the immunoregulatory activity of galectin-1: Control of immune cell trafficking, dendritic cell physiology, and T-cell fate. Methods Enzymol. 2010;480:199-244.

36. Fitzner B, Walzel H, Sparmann G, Emmrich J, Liebe S, Jaster R. Galectin-1 is an inductor of pancreatic stellate cell activation. Cell Signal. 2005;17(10):1240-7.

37. Chung JC, Oh MJ, Choi SH, Bae CD. Proteomic analysis to identify biomarker proteins in pancreatic ductal adenocarcinoma. ANZ J Surg. 2008;78(4):245-51.

38. Kuramitsu Y, Taba K, Ryozawa S, Yoshida K, Zhang X, Tanaka T, et al. Identification of up- and down-regulated proteins in gemcitabine-resistant pancreatic cancer cells using two-dimensional gel electrophoresis and mass spectrometry. Anticancer Res. 2010;30(9):3367-72.

39. Chen R, Pan S, Ottenhof NA, de Wilde RF, Wolfgang CL, Lane Z, et al. Stroma galectin-1 expression is associated with long-term survival in resectable pancreatic ductal adenocarcinoma. Cancer Biol Ther. 2012:13(10):899-907.

40. Yamato I, Sho M, Nomi T, Akahori T, Shimada K, Hotta K, et al. Clinical importance of B7-H3 expression in human pancreatic cancer. $\mathrm{Br} \mathrm{J}$ Cancer. 2009;101(10):1709-16. 
41. Yamamoto K, Ueno T, Kawaoka T, Hazama S, Fukui M, Suehiro Y, et al. MUC1 peptide vaccination in patients with advanced pancreas or biliary tract cancer. Anticancer Res. 2005;25(5):3575-9.

42. Ramanathan RK, Lee KM, McKolanis J, Hitbold E, Schraut W, Moser AJ, et al. Phase I study of a MUC1 vaccine composed of different doses of MUC1 peptide with SB-AS2 adjuvant in resected and locally advanced pancreatic cancer. Cancer Immunol Immunother. 2005;54(3):254-64.

43. Petrulio CA, Kaufman HL. Development of the PANVAC-VF vaccine for pancreatic cancer. Expert Rev Vaccines. 2006;5(1):9-19.

44. Van Cutsem E, Vervenne WL, Bennouna J, Humblet Y, Gill S, Van Laethem $J$, et al. Phase III trial of bevacizumab in combination with gemcitabine and erlotinib in patients with metastatic pancreatic cancer. J Clin Oncol. 2009;27(13):2231-7.

45. Kindler HL, Niedzwiecki D, Hollis D, Sutherland S, Schrag D, Hurwitz H, et al. Gemcitabine plus bevacizumab compared with gemcitabine plus placebo in patients with advanced pancreatic cancer: phase III trial of the Cancer and Leukemia Group B (CALGB 80303). J Clin Oncol. 2010;28(22):3617-22.

46. Yamaue H, Tani T, Miyazawa M, Yamao K, Mizuno N, Okusaka T, et al. Phase II/III clinical trial with VEGFR2-epitope peptide and gemcitabine for patients with locally advanced, metastatic, or unresectable pancreatic cancer: Pegasus-PC study. J Clin Oncol. 2013;4:abstr 223.

47. Middleton G, Silcocks P, Cox T, Valle J, Wadsley J, Propper D, et al. Gemcitabine and capecitabine with or without telomerase peptide vaccine GV1001 in patients with locally advanced or metastatic pancreatic cancer (TeloVac): an open-label, randomised, phase 3 trial. Lancet Oncol. 2014;15(8):829-40.

48. Schmidt J. Pharmexa Stops One of Two Phase III Trials. 2008; Available at: http:/ www.drugs.com/news/pharmexa-stops-one-two-phase-iii-trials-8180.html, 2014 Accessed 16 Feb 2016.

49. Buanes T, Maurel J, Liauw W, Hebbar M, Nemunaitis J. A randomized phase III study of gemcitabine (G) versus GV1001 in sequential combination with $\mathrm{G}$ in patients with unresectable and metastatic pancreatic cancer (PC) J Clin Oncol (Meeting Abstracts) May 2009;27(no. 15S 4601).

50. Bernhardt SL, Gjertsen MK, Trachsel S, Moller M, Eriksen JA, Meo M, et al. Telomerase peptide vaccination of patients with non-resectable pancreatic cancer: A dose escalating phase I/II study. Br J Cancer. 2006;95(11):1474-82.

51. Gilliam AD, Broome P, Topuzov EG, Garin AM, Pulay I, Humphreys J, et al. An international multicenter randomized controlled trial of G17DT in patients with pancreatic cancer. Pancreas. 2012:41(3):374-9.

52. Brett BT, Smith SC, Bouvier CV, Michaeli D, Hochhauser D, Davidson BR, et al. Phase II study of anti-gastrin-17 antibodies, raised to G17DT, in advanced pancreatic cancer. J Clin Oncol. 2002;20(20):4225-31.

53. Shapiro J, Marshall J, Karasek P, et al. G17DT + gemcitabine versus placebo + gemcitabine in untreated subjects with locally advanced, recurrent or metastatic adenocarcinoma of the pancreas: results of a randomized, doubleblinded, multinational, multicenter study. J Clin Oncol. 2005;23(suppl):4012.

54. Gjertsen MK, Buanes T, Rosseland AR, Bakka A, Gladhaug I, Soreide O, et al. Intradermal ras peptide vaccination with granulocyte-macrophage colonystimulating factor as adjuvant: Clinical and immunological responses in patients with pancreatic adenocarcinoma. Int J Cancer. 2001;92(3):441-50.

55. Abou-Alfa GK, Chapman PB, Feilchenfeldt J, Brennan MF, Capanu M, Gansukh B, et al. Targeting mutated K-ras in pancreatic adenocarcinoma using an adjuvant vaccine. Am J Clin Oncol. 2011;34(3):321-5.

56. Weden S, Klemp M, Gladhaug IP, Moller M, Eriksen JA, Gaudernack G, et al. Long-term follow-up of patients with resected pancreatic cancer following vaccination against mutant K-ras. Int J Cancer. 2011:128(5):1120-8.

57 Muscarella P et al. A randomized, placebo controlled, doubled, blind, multicenter phase 2 adjuvant trial of the efficacy, immunogenicity, and safety of Gl-4000 plus Gem vs Gem alone in patients with resected pancreas cancer with activating Ras mutations/survival and immunology analysis of the R1 Subgroup. J Clin Oncol. 2012;30(suppl):astr \#e14501.

58. Kondo H, Hazama S, Kawaoka T, Yoshino S, Yoshida S, Tokuno K, et al. Adoptive immunotherapy for pancreatic cancer using MUC1 peptide-pulsed dendritic cells and activated T lymphocytes. Anticancer Res. 2008;28(1B):379-87.

59. Kimura Y, Tsukada J, Tomoda T, Takahashi H, Imai K, Shimamura K, et al. Clinical and immunologic evaluation of dendritic cell-based immunotherapy in combination with gemcitabine and/or S-1 in patients with advanced pancreatic carcinoma. Pancreas. 2012;41(2):195-205.

60. Jaffee EM, Hruban RH, Biedrzycki B, Laheru D, Schepers K, Sauter PR, et al. Novel allogeneic granulocyte-macrophage colony-stimulating factorsecreting tumor vaccine for pancreatic cancer: a phase I trial of safety and immune activation. J Clin Oncol. 2001;19(1):145-56.
61. Lutz E, Yeo CJ, Lillemoe KD, Biedrzycki B, Kobrin B, Herman J, et al. A lethally irradiated allogeneic granulocyte-macrophage colony stimulating factorsecreting tumor vaccine for pancreatic adenocarcinoma. A Phase II trial of safety, efficacy, and immune activation. Ann Surg. 2011;253(2):328-35.

62. Laheru D, Lutz E, Burke J, Biedrzycki B, Solt S, Onners B, et al. Allogeneic granulocyte macrophage colony-stimulating factor-secreting tumor immunotherapy alone or in sequence with cyclophosphamide for metastatic pancreatic cancer: a pilot study of safety, feasibility, and immune activation. Clin Cancer Res. 2008;14(5):1455-63.

63. Le DT, Wang-Gillam A, Picozzi V, Greten TF, Crocenzi T, springett G, et al. Safety and survival with GVAX pancreas prime and Listeria Monocytogenesexpressing mesothelin (CRS-207) boost vaccines for metastatic pancreatic cancer. J Clin Oncol. 2015;33(12):1325-33.

64. Kawaoka T, Oka M, Takashima M, Ueno T, Yamamoto K, Yahara N, et al. Adoptive immunotherapy for pancreatic cancer: cytotoxic $T$ lymphocytes stimulated by the MUC1-expressing human pancreatic cancer cell line YPK-1. Oncol Rep. 2008;20(1):155-63.

65. Beatty GL. Safety and antitumor activity of chimeric antigen receptor modified T cells in patients with chemotherapy refractory metastatic pancreatic cancer. 2015 ASCO Annual Meeting; 2015.

66. Hodi FS, O'Day SJ, McDermott DF, Weber RW, Sosman JA, Haanen JB, et al. Improved survival with ipilimumab in patients with metastatic melanoma. N Engl J Med. 2010;363(8):711-23.

67. Robert C, Long GV, Brady B, Dutriaux C, Maio M, Mortier L, et al. Nivolumab in previously untreated melanoma without BRAF mutation. N Engl J Med. 2015;372(4):320-30

68. Beatty GL, Chiorean EG, Fishman MP, Saboury B, Teitelbaum UR, Sun W, et al. CD40 agonists alter tumor stroma and show efficacy against pancreatic carcinoma in mice and humans. Science. 2011;331(6024):1612-6.

69. Brahmer JR, Tykodi SS, Chow LQ, Hwu WJ, Topalian SL, Hwu P, et al. Safety and activity of anti-PD-L1 antibody in patients with advanced cancer. N Engl J Med. 2012;366(26):2455-65.

70. Royal RE, Levy C, Turner K, Mathur A, Hughes M, Kammula US, et al. Phase 2 trial of single agent Ipilimumab (anti-CTLA-4) for locally advanced or metastatic pancreatic adenocarcinoma. J Immunother. 2010;33(8):828-33.

71. Segal NH, Anto SJ, Brahmer JR, et al. Preliminary data from a multi-arm expansion study of MEDI4736, an anti-PD-L1 antibody. J Clin Oncol. 2014;32(5 s):abstr 3002.

72. Plate JM, Plate AE, Shott S, Bograd S, Harris JE. Effect of gemcitabine on immune cells in subjects with adenocarcinoma of the pancreas. Cancer Immunol Immunother. 2005;54(9):915-25.

73. Correale P, Cusi MG, Del Vecchio MT, Aquino A, Prete SP, Tsang KY, et al. Dendritic cell-mediated cross-presentation of antigens derived from colon carcinoma cells exposed to a highly cytotoxic multidrug regimen with gemcitabine, oxaliplatin, 5-fluorouracil, and leucovorin, elicits a powerful human antigen-specific CTL response with antitumor activity in vitro. J Immunol. 2005;175(2):820-8.

74. Kershaw MH, Devaud C, John LB, Westwood JA, Darcy PK. Enhancing immunotherapy using chemotherapy and radiation to modify the tumor microenvironment. Oncoimmunology. 2013;2(9):e25962.

75. Le DT, Lutz E, Uram JN, Sugar EA, Onners B, Solt S, et al. Evaluation of ipilimumab in combination with allogeneic pancreatic tumor cells transfected with a GM-CSF gene in previously treated pancreatic cancer. J Immunother. 2013;36(7):382-9.

76. Le DT, Uram JN, Wang H, Bartlett BR, Kemberling H, Eyring AD, et al. PD-1 Blockade in Tumors with Mismatch-Repair Deficiency. N Engl J Med. 2015; 372(26):2509-20.

77. Geynisman DM, Zha Y, Kunnavakkam R, Aklilu M, Catenacci DV, Polite BN, et al. A randomized pilot phase I study of modified carcinoembryonic antigen (CEA) peptide (CAP1-6D)/montanide/GM-CSF-vaccine in patients with pancreatic adenocarcinoma. J Immunother Cancer. 2013;1:8-1426-1-8. eCollection 2013.

78. Schuetz T, Kaufman H, Marshall J, Safran H. Extended survival in second-line pancreatic cancer after therapeutic vaccination.2005. 16 suppl 2576. ASCO Annual Meeting.

79. Jesus-Acosta A, O'Dwyer P, Ramanathan R, et al. A phase II study of vismodegib, a hedgehog $(\mathrm{Hh})$ pathway inhibitor, combined with gemcitabine and nab-paclitaxel (nab-P) in patients (pts) with untreated metastatic pancreatic ductal adenocarcinoma (PDA). J Clin Oncol 32, 2014 (suppl 3; abstr 257). ASCO Annual Meeting.

80. Starodub A, Ocean A, Shah M, et al. SN-38 antibody-drug conjugate (ADC) targeting Trop-2, IMMU-132, as a novel platform for the therapy of diverse metastatic solid cancers: Initial clinical results. J Clin Oncol 32:5s, 2014 (suppl; abstr 3032). ASCO Annual Meeting 
81. Nishida S, Koido S, Takeda Y, Homma S, Komita H, Takahara A, et al. Wilms tumor gene (WT1) peptide-based cancer vaccine combined with gemcitabine for patients with advanced pancreatic cancer. J Immunother. 2014:37(2):105-14.

82. Nakamura M, Wada J, Suzuki H, Tanaka M, Katano M, Morisaki T. Long-term outcome of immunotherapy for patients with refractory pancreatic cancer. Anticancer Res. 2009;29(3):831-6.

83. Lepisto AJ, Moser AJ, Zeh H, Lee K, Bartlett D, McKolanis JR, et al. A phase I/ II study of a MUC1 peptide pulsed autologous dendritic cell vaccine as adjuvant therapy in patients with resected pancreatic and biliary tumors. Cancer Ther. 2008;6(B):955-64.

84. Pecher G, Haring A, Kaiser L, Thiel E. Mucin gene (MUC1) transfected dendritic cells as vaccine: results of a phase I/II clinical trial. Cancer Immuno Immunother. 2002;51(11-12):669-73.

85. Koido S, Homma S, Okamoto M, Mori M, and Yoshizaki S. Dendritic cells pulsed with Wilms' tumor gene 1 (WT1)-specific and MHC class I and IIrestricted epitopes with gemcitabine induce antitumor immune responses in patients with advanced pancreatic cancer. Cancer Research. October 1, 2014;74:CT223. AACR Annual Meeting.

\section{Submit your next manuscript to BioMed Central} and we will help you at every step:

- We accept pre-submission inquiries

- Our selector tool helps you to find the most relevant journal

- We provide round the clock customer support

- Convenient online submission

- Thorough peer review

- Inclusion in PubMed and all major indexing services

- Maximum visibility for your research

Submit your manuscript at www.biomedcentral.com/submit 